\title{
2016 Levi L. Conant Prize
}

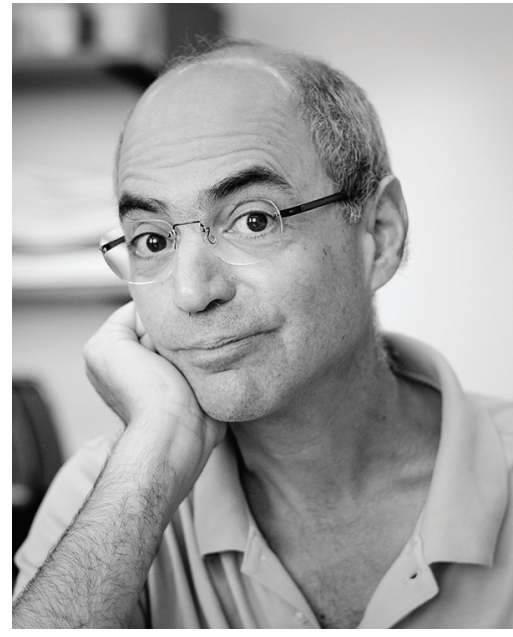

Daniel Rothman
DANIEL ROTHMAN was awarded the 2016 Levi L. Conant Prize at the 122nd Annual Meeting of the AMS in Seattle, Washington, in January 2016.

\section{Citation}

The 2016 Levi L. Conant Prize is awarded to Daniel Rothman for his article "Earth's carbon cycle: A mathematical perspective", which appeared in the Bulletin of the American Mathematical Society 52 (2015), no. 1, 47-64.

Mathematics finds a place in science by enunciating principles that at once classify, quantify, and illuminate natural phenomena. This heuristic is often best displayed when phenomena are simple or at least isolated from external influences. In this sense, biological processes present a particular challenge for mathematics because they are generally tapestries of confounding factors. It is nowadays common to approach such problems from a viewpoint that promotes data collection and data analysis as the pathway to knowledge. But, as Dan Rothman points out in this article, "Data, however, require understanding." Here, he gives us an understanding of the Earth's carbon cycle by applying classical ideas from applied mathematics to the data at hand.

The carbon cycle of the Earth uses plant photosynthesis to combine carbon dioxide and water to produce carbohydrates and free oxygen. To complete the cycle, respiration uses carbohydrates and free oxygen to give carbon dioxide and water. However, there is a leak in this process. Some carbohydrates become trapped in rock and this entails

For permission to reprint this article, please contact: reprint-permission@ams.org.

DOI: http://dx.doi.org/10.1090/noti1353 a surplus of free oxygen. Thus our atmosphere is born; life as we know it is a consequence of a feedback error. Rothman writes, "Earth's carbon cycle, therefore, plays a major role in determining not only [carbon dioxide] levels and climate, but also life's interaction with the physical environment."

Mathematics informs our understanding of the carbon cycle in a variety of ways. A fundamental part of the cycle involves the decay of organic matter. On the face of it, this is an intractable problem because organic matter comes in a multitude of forms, each with its own decay parameter. This type of disordered kinetics may be studied, however, by considering the probability density function of decay parameters and showing that decay is characterized by the Laplace transform of this density function. Data then informs the mathematics by suggesting that the density function is lognormal and, indeed, that plant decay always has lognormal density parameters. As the author says, this seems to be a "natural manifestation" of the Central Limit Theorem. Once the ubiquity of lognormality is established as an underlying principle, it allows calculations such as mean-time-todecay which allow us to compare data with theory. Rothman goes on to discuss the scaling factors that arise in consideration of decay on land, sea, or in sediments, and this leads to his conclusion that respiration follows the "rhythm of a logarithmic clock" where local microorganisms, acting on biological time scales, have a global influence on geological time scales. Rothman also gives us an understanding of long-term fluctuations in carbon dioxide levels in the atmosphere, as well as the long-term immobilization of organic carbon in rock, the latter eliciting the question of how free oxygen levels could have ever increased from a stable state. This is precisely what good mathematical models do: they explain some phenomena and they raise questions about others.

This article is especially timely as humanity grapples with the consequences of releasing trapped carbon by burning fossil fuels. The author, a geophysicist, concludes with an invitation to mathematicians to take up the challenge: 
"Because the carbon cycle represents the coupling between life and the environment-metabolism at a global scale-its mathematical description inherits the difficulties of biology in addition to physical science. Thus, theoretical understanding of dynamics, so crucial to advancing knowledge of how the carbon cycle works, remains more qualitative than quantitative. Such problems present scientific opportunities with no shortage of social significance. Mathematics will surely play a central role in future progress."

But it isn't just the timeliness of the topic that draws us in and holds our interest. At every step the author takes care to explain the science as well as the mathematics involved in clear straightforward prose so that the entire article is accessible to a general mathematical audience. We often hear about Wigner's "unreasonable effectiveness of mathematics in the sciences," but it is not so often that we see this manifested in a beautiful exposition of a fundamental ingredient of our existence.

\section{Biographical Sketch}

Daniel H. Rothman received his undergraduate degree in applied mathematics from Brown University in 1979 and his $\mathrm{PhD}$ in geophysics from Stanford University in 1986 . He is currently a professor of geophysics in the Department of Earth, Atmospheric, and Planetary Sciences at the Massachusetts Institute of Technology, where he has been on the faculty since 1986. In 2011 he cofounded the Lorenz Center, an interdisciplinary research center at MIT devoted to developing the foundations of climate science. Rothman was a Fellow of the Radcliffe Institute of Advanced Study in 2007-08. He has also been honored as a fellow of the American Physical Society (2012) and the American Geophysical Union (2014). His research spans several fields-including fluid mechanics, complex systems, and earth-system dynamics-in which he uses methods of applied mathematics and statistical physics to learn how the natural environment works.

\section{Response from Daniel Rothman}

I am delighted and honored to receive the 2016 Levi L. Conant Prize for my article in the Bulletin of the AMS. The paper was written to complement my invited talk for the Current Events Bulletin at the 2014 Joint Mathematics Meetings. I would like to thank David Eisenbud for the invitation and his encouragement of my subject. Susan Friedlander's enthusiastic support as editor of the Bulletin is also much appreciated. I'm thrilled that the talk and the resulting paper met such an appreciative audience. The carbon cycle resides at the intersection of biology, geology, and chemistry. Finding my way through the convergence of these subjects was like learning French: initially little made sense, but with knowledge and experience a logical structure began to emerge. I never could have arrived at that point without the help of others. I'm especially thankful that my MIT colleagues Eric Alm, Tanja Bosak, Sam Bowring, Ed Boyle, Greg Fournier, and Roger Summons have found my efforts worthy of collaboration. My students Chris Follett, David Forney, and Alex Petroff also helped guide me through the complexity of the subject. None of this would have been possible without the patient tutelage of John Hayes. During four summer visits to Woods Hole, I learned the intricacies of the carbon cycle from a master. I am deeply grateful that John recognized potential in my early forays into the field.

There may be no better way to secure our collective future than to understand more deeply the natural world around us. A significant portion of that understanding will derive from insightful applications of mathematics. I thank the Conant Prize Selection Committee and the American Mathematical Society for recognizing the intrinsic value of such work.

\section{About the Prize}

The Levi L. Conant Prize is awarded annually to recognize an outstanding expository paper published in either the Notices of the AMS or the Bulletin of the AMS in the preceding five years.

Established in 2001, the prize honors the memory of Levi L. Conant (1857-1916), who was a mathematician at Worcester Polytechnic Institute. The prize carries a cash award of US $\$ 1,000$.

The Conant Prize is awarded by the AMS Council acting on the recommendation of a selection committee. For the 2016 prize, the members of the selection committee were the following individuals.

- John C. Baez

- Carolyn Gordon

- John F. Oprea (Chair)

The complete list of recipients of the Levi L. Conant Prize follows.

2001 Carl Pomerance

2002 Elliott Lieb, Jakob Yngvason

2003 Nicholas Katz, Peter Sarnak

2004 Noam D. Elkies

2005 Allen Knutson, Terence Tao

2006 Ronald M. Solomon

2007 Jeffrey Weeks

2008 J. Brian Conrey, Shlomo Hoory,

Nathan Linial, Avi Wigderson

2009 John W. Morgan

2010 Bryna Kra

2011 David Vogan

2012 Persi Diaconis

2013 John Baez, John Huerta

2014 Alex Kontorovich

2015 Jeffrey C. Lagarias, Chuanming Zong

2016 Daniel Rothman 